\section{Structure of Selenium Tetrafluoride}

IN his interesting communication on the structure of $\mathrm{SeF}_{4}{ }^{1}$, Mr. Bowen writes: "Only one model was found to give satisfactory agreement with the visually estimated ring intensities. This model has a distorted tetrahedron of fluorine atoms around the selenium atom with two opposite FSeF angles of $120^{\circ}$ and the remaining four FSeF angles equal to $104 \cdot 5^{\circ} \ldots$ the uncertainty in the angles [is] $\pm 10^{\circ} \%$.

Now the relationship between the six internal angles in a distorted tetrahedron the corners of which remain equidistant from the centre is

$\cos ^{2} \frac{\alpha}{2}=-\cos \beta$, or, $\cos \alpha+2 \cos \beta=-1$,

where $\alpha$ is one of the two equal angles, while $\beta$ is one of the four equal angles. For $\alpha=120^{\circ}$ we have $\beta=104^{\circ} 29^{\prime}$, in agreement with $\mathrm{Mr}$. Bowen's results. The $\alpha$ values of $110^{\circ}$ and $130^{\circ}$ yield, however, $109^{\circ}$ $13^{\prime}$ and $100^{\circ} 17^{\prime}$ for $\beta$, respectively. Hence $120^{\circ} \pm$ $10^{\circ}$ for $\alpha$ corresponds with $104^{\circ} 29^{\prime} \pm$ c. $4^{\circ} 30^{\prime}$ for $\beta$.

It may be of interest to note that, while the analogous six angles in a regular tetrahedron are equal to $109^{\circ} 28^{\prime}=\sec ^{-1}(-3)$, those quoted by Mr. Bowen are $\sec ^{-1}(-2)=120^{\circ}$ and $\sec ^{-1}(-4)=$ $104^{\circ} 29^{\prime}$. I should like to add that angles formed by the maximum number of equally spaced radii with a common origin are equal to $\sec ^{-1}(-1)$, $\sec ^{-1}(-2)$ and $\sec ^{-1}(-3)$, for the uni-, bi- and terdimensional space, respectively.

Commonwealth Forestry Bureau,

Forest Products Research Laboratory,

Princes Risborough, Bucks. July 27.

Nature, 171, 171 (1953).

\section{Separation of Racemic Mixtures by Electrophoresis in a Strong Centrifugal or Magnetic Field}

THE separation of racemic mixtures of optical isomers is still limited, with rare exceptions, to the methods of Pasteur. For this reason the resolution of substances which seem identical in the test-tube but are importantly different in biology usually proves to be difficult and expensive both in research and in the chemical industry.

A theory for a generic method must be stated in terms of a gross model. The derivation of equations for the movement of a body in an electromagnetic field becomes almost prohibitively difficult except in the case of a sphere or an ellipsoid, as Alexander Kolin ${ }^{1}$ has found in his study of the migration of visible particles. Furthermore, the molecules of water are far from infinitesimal in size when compared with the important racemic substances. Since the solvent is not a 'uniform homogeneous fluid', any application of the theorems of hydrodynamics will have at best only qualitative validity.

Consider a racemic mixture- $d$ - and $l$-phenylalanine, for example-in a solution which will cause ionization as an acid. Suppose a low-voltage electrophoretic current to flow through the solution, and a strong centrifugal or a non-homogeneous magnetic field to be at right-angles to the direction of molecular migration.

A simple model may be pictured as follows. One may imagine a large number of small boats drifting on a lake, the boats being identical excepting that half have their rudders permanently fixed ten degrees to the right, and the other half ten degrees to the left. Let us further imagine a light wind in a given direction. The boats which were floating at random will tend to be turned with their prows in the same direction as the wind, since they can move in this direction with least resistance. One may ordinarily picture the two sets of boats as diverging at a constant angle according to the setting of the rudders. If there should be sufficient increase in momentum, however, the angular departure from the direction of the wind would increase until the boats are broadside to the wind. Here they will again be oriented with the wind after making a loop or node. In either case, the two groups of boats will travel with an increasing separation to the right and to the left from the direction of the wind.

This physical model corresponds to the specifications stated for the amino-acid mixture. The electrophoretic current corresponds to the wind, the ionization as an acid furnishes the prow and stern polarity, and the asymmetry of the molecules corresponds to the fixation of the rudders to the right or to the left. Finally, the action of the magnetic field upon the most strongly diamagnetic part of the molecule, or else of the centrifugal field upon the part of the molecule with greatest density, will provide the equivalent of a keel with a constant downward direction in the lake.

A sharp resolution, of course, would require the use of a solvent that will minimize the disturbance of Brownian movement. This can be achieved by cooling the water to the point of maximum density, or, in appropriate cases, by using a solvent which remains liquid at a much lower temperature.

College of Medicine,

University of Illinois. Chicago 12.

1 Science, 117, 134 (1953). Frederick C. LeNDRUM

\section{The Delhi Pillar}

The freedom from rusting of the famous iron pillar at Delhi has long been a subject of comment and has been attributed by some writers to the peculiar properties of the ancient iron from which it is forged. Although some particulars of its history are obscure, it seems certain that the pillar dates from about the fifth century A.D, and is roughly 1,500 years old. The immunity of iron from rusting over such a long period is a striking phenomenon and it may therefore be of interest to report the results of some experiments that were made to ascertain the reason for this.

Table 1. ATMOSPhERIO CoRrosion OF STEFI AND ZINO

\begin{tabular}{|c|c|c|c|}
\hline \multirow{2}{*}{\multicolumn{2}{|c|}{ Exposure station }} & \multicolumn{2}{|c|}{$\begin{array}{l}\text { Corrosion rate. Mils }(0 \cdot 001 \text { in.) } \\
\text { per year }\end{array}$} \\
\hline & & Steel* & Zinc \\
\hline $\begin{array}{l}\text { Delhi } \\
\text { Godalming } \\
\text { Sheffeld } \\
\text { Khartoum } \\
\text { Basrah } \\
\text { Singapore }\end{array}$ & 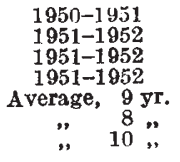 & $\begin{array}{l}0 \cdot 23 \\
0 \cdot 17 \\
1 \cdot 77 \\
4 \cdot 2 \\
0 \cdot 1 \\
0 \cdot 6 \\
0 \cdot 6\end{array}$ & $\begin{array}{l}0 . \overline{006} \\
0 \cdot 042 \\
0 \cdot 51 \\
0.02 \\
0 \cdot 04 \\
0 \cdot 04\end{array}$ \\
\hline
\end{tabular}

- The specimens exposed at Delhi, Godalming and Sheffield were of steel containing $0 \cdot 3$ per cent of copper. Thls material ts roughly onequarter less corrodible than ingot iron, of which the Khartoum, Basrah and Singapore specimens were made. 